\title{
MARKOV CHAINS WITH \\ QUASITOEPLITZ TRANSITION MATRIX: \\ FIRST ZERO HITTING
}

\author{
Alexander M. Dukhovny \\ Department of Mathematics \\ San Francisco State University \\ San Francisco, CA 94132
}

\begin{abstract}
This paper continues the investigation of Markov Chains with a quasitoeplitz transition matrix. Generating functions of first zero hitting probabilities and mean times are found by the solution of special Riemann boundary value problems on the unit circle. Duality is discussed.
\end{abstract}

AMS Subject Classification: 60J, 60K25.

Key Words: Markov chains, quasitoeplitz matrix, zero hitting, Riemann boundary value problem, duality.

\section{INTRODUCTION.}

Here we continue the investigation started in [1] of Markov chains with a quasitoeplitz transition matrix, elements of which depend on the difference of indices except for the first column and the first several rows. The primary focus of this paper is the problem of first zero hitting (FZH) in terms of generating functions (GF) of probabilities and mean times.

The practical importance of this problem is in its obvious connection with the problems of first emptying and response time arising in applications. There is also another reason for investigation: duality of this problem and state probabilities evaluation.

The FZH problem was only studied in that simplest particular case

\footnotetext{
${ }^{0}$ Received: July 1989, Revised: August 1989
} 
when the transition matrix was in fact toeplitz (except, of course, in the first column). In this case FZH is in fact a problem of the first entering nonpositive area for some random walk on the integers, so relevant GF's were found either in a purely probabilistic manner (see, for example, [2]), or by the solution of a Riemann boundary value problem (for example, Dukhovny [3] for a random walk that depends on a parameter).

In this paper we study a quasitoeplitz case arising in applications with feedback, so there is no structure of a random walk at some early states. We transform the FZH problem to nonuniform Riemann boundary value problems for the GF's of probabilities and mean times. As the sums of these do not converge, we deal in fact with boundary problems in a wide sense, that is in special Banach spaces (see [4]).

We also prove here that FZH mean times do not exceed some linear function of a starting state's number.

Having solved the boundary value problems, we obtain some finite systems of linear equations and prove that they have only one solution.

In conclusion we discuss a duality between transient and steady state probabilities from one side, and FZH probabilities and mean times' increments, respectively, from the other, imposed by the method of boundary value problems.

\section{DEFINITIONS.}

We assume here that we have a Markov chain $\left\{\xi_{k}\right\}$ with a quasitoeplitz transition matrix $A=\left(a_{i j}\right)$ with "natural" properties (see [1], pp. 72-73). We retain the notation of [1] for $A_{i}(z)=\sum_{j=0}^{\infty} a_{i j} z^{j}, H(z)=\sum_{-\infty}^{\infty} h_{j} z^{j}$, level $n$ such that $\forall i \geq n a_{i j}=h_{j-i}, j>0 ; a_{i 0}=\sum_{-\infty}^{-i} h_{j}$; operatores $T^{+}$ and $T^{-}$that cut out parts with nonnegative and negative exponents of Laurent power series, $D^{i}$ that gets the $i$ th coefficient of the series, space $L_{1}$ of Laurent power series with absolutely summable coefficients, unit circle $\Gamma:|z|=1$, its interior $\Gamma^{+}$and exterior $\Gamma^{-}$.

Let us introduce $q_{i}^{k}, i>0, k>0$, the probability of reaching zero for the first time in $k$ steps starting from $i$, and $\mu_{i}=\sum_{k=1}^{\infty} k q_{i}^{k}$, the mean number of steps in the FZH route starting from $i$, which is known to converge if the chain is ergodic. We also denote $q_{i}^{0}=-1, i>0$. 
Let $x \in(0,1),|z|>1$, and denote

$$
\begin{aligned}
q_{i}(x) & =\sum_{k=0}^{\infty} q_{i}^{k} x^{k}, \\
\mathbf{Q}(\mathbf{x}) & =\left(0, q_{1}(x), q_{2}(x), \ldots\right)^{T}, \\
Q(x, z) & =\sum_{i=1}^{\infty} q_{i}(x) z^{-i} \\
\mu(z) & =\sum_{i=1}^{\infty} \mu_{i} z^{-i} .
\end{aligned}
$$

We introduce the linear Banach space $L_{\infty}$ of formal Laurent power series with bounded coefficients with a norm

$$
\left\|\sum_{i=-\infty}^{\infty} a_{i} z^{i}\right\|=s u p_{i}\left|a_{i}\right|
$$

and a formal product of series (convolution)

$$
a(z) b(z)=\sum_{i=-\infty}^{\infty} z^{i} \sum_{j=-\infty}^{\infty} a_{i-j} b_{j}
$$

provided the absolute convergence of sums

$$
\sum_{j=-\infty}^{\infty} a_{i-j} b_{j}, \quad \forall i
$$

Note that if $a(z) \in L_{1}$ and $b(z) \in L_{\infty}$ then their convolution obviously belongs to $L_{\infty}$.

Whatever $i$ is, a total FZH probability $\sum_{k=1}^{\infty} q_{i}^{k}$ does not exceed 1, so

$$
-1=q_{i}(0) \leq q_{i}(x) \leq q_{i}(1) \leq 0 \quad x \in[0,1]
$$

hence $Q(x, z) \in L_{\infty}$.

Denote $e(z)=\sum_{i=1}^{\infty} z^{-i} \in L_{\infty}$.

Lemma 1. Let $a^{+}(z)=\sum_{i=0}^{\infty} a_{i} z^{i} \in L_{1}$. Then $T^{-} a^{+}(z) e(z)=a^{+}(1) e(z)$.

Proof. According to the definitions of $T^{-}$and the convolution

$$
T^{-} a^{+}(z) e(z)=\sum_{i=-\infty}^{-1} z^{i} \sum_{j=-\infty}^{\infty} a_{i-j} D^{j} e(z)=\sum_{i=-\infty}^{-1} z^{i} \sum_{j=0}^{\infty} a_{j}=a^{+}(1) e(z) .
$$




\section{FIRST ZERO HITTING PROBABILITIES.}

Every FZH route from $i>0$ is either a direct step to zero or consists of one step to some $j>0$ and a FZH route from $j$, so

$$
q_{i}^{k+1}=\sum_{j=1}^{\infty} a_{i j} q_{j}^{k} \quad k \geq 0, i>0 .
$$

Multiplying both sides by $x^{k+1}$ and summing over $k$ from 0 to $\infty$ we obtain

$$
q_{i}(x)=x \sum_{j=1}^{\infty} a_{i j} q_{j}(x)+(x-1) \quad i>0
$$

or, in vector form,

$$
\mathbf{Q}(\mathbf{x})=x A^{1} \mathbf{Q}(\mathbf{x})+(x-1) \mathbf{1}
$$

where $1=(0,1,1, \ldots)$, and $A^{1}$ differs from $A$ only by elements of the first row and column, which are all zeros except $a_{00}^{1}=1$.

Proceeding from (4) we now find the GF $Q(x, z)$.

Theorem 1. The GF $Q(x, z), x \in(0,1),|z|>1$, of the first zero hitting probabilities of the Markov chain $\left\{\xi_{k}\right\}$ with a quasitoeplitz transition matrix with $n>0$ is defined by the following formulas:

$$
\begin{aligned}
Q(x, z)= & z^{1-n} R^{-}(x, z) \\
& {\left[(x-1) R^{+}(x, 1) e(z)+\sum_{i=1}^{n-1} q_{i}(x) T^{+} z^{n-1-i} R^{-}(x, z)^{-1}\right] }
\end{aligned}
$$

(6) $R^{ \pm}(x, z)=\exp \left(-T^{ \pm} \ln [1-x H(z)]\right)$

and the unknowns $q_{i}(x), i=1,2, \ldots, n-1$ are the only solution of the system of linear equations

$$
\begin{aligned}
& q_{i}(x)-x \sum_{j=1}^{n-1} q_{j}(x) D^{n-1} A_{i}(z) R^{-}(x, z) T^{+} z^{n-1-j} R^{-}(x, z) \\
& =(x-1)\left[1+x R^{+}(x, 1) D^{n-1} A_{i}(z) R^{-}(x, z) e(z)\right] \quad i=1,2, \ldots, n-1
\end{aligned}
$$

( $n=0$ and $n=1$ obviously generate the same FZH problems). 
Proof. According to the definitions made

$$
\sum_{j=1}^{\infty} a_{i j} q_{j}(x)= \begin{cases}D^{0} A_{i}(z) Q(x, z) & i<n \\ D^{-i} H(z) Q(x, z) & i \geq n .\end{cases}
$$

Note that the convolutions $A_{i}(z) Q(x, z)$ and $H(z) Q(x, z)$ exist and belong to $L_{\infty}$ as $A_{i}(z)$ and $H(z)$ by definition belong to $L_{1}$. Therefore, the subset of equalities (3) for $i \geq n$ yields

$$
Q_{2}(x, z)=x T^{-} H(z)\left[Q_{2}(x, z)+z^{n-1} Q_{1}(x, z)\right]+(x-1) e(z)
$$

where

$$
\begin{aligned}
& Q_{1}(x, z)=\sum_{i=1}^{n-1} q_{i}(x) z^{-1} \\
& Q_{2}(x, z)=\sum_{i=n}^{\infty} q_{i}(x) z^{n-1-i} .
\end{aligned}
$$

We transform (9) to the formal equality of power series

$$
\begin{aligned}
& Q_{2}(x, z) \sum_{i=1}^{n-1}[1-x H(z)] \\
& =F^{+}(x, z)+(x-1) e(z)-[1-x H(z)] z^{n-1} Q_{1}(x, z)
\end{aligned}
$$

where

$$
F^{+}(x, z)=-x T^{+} H(z) Q_{2}(x, z)+T^{+}[1-x H(z)] z^{n-1} Q_{1}(x, z) .
$$

In (10) the power series $Q_{2}(x, z)$ and $F^{+}(x, z)$ correspond to functions analytic in $\Gamma^{-}$and $\Gamma^{+}$, respectively; but they need not converge on $\Gamma$, so the equality (10) is the formal equality of the power series, not their sums. Therefore (10) must be treated as a Riemann boundary value problem in a wide sense.

Let us multiply (10) by $R^{+}(x, z)$ and apply $T^{-}$to both sides. Due to (6) on $\Gamma:|z|=1, R^{+}(x, z) R^{-}(x, z)=[1-x H(z)]^{-1}$, so with the help of Lemma 1 we obtain:

$$
Q_{2}(x, z) R^{-}(x, z)^{-1}=(x-1) R^{+}(x, 1) e(z)-\sum_{i=1}^{n-1} q_{i}(x) T^{-} z^{n-1-i} R^{-}(x, z)^{-1},
$$


which yields (5).

To obtain (7) we transform some first equalities of (3) as follows:

$$
q_{i}(x)=x D^{0} A_{i}(z) Q(x, z)+(x-1) \quad i=1,2, \ldots, n-1
$$

and substitute (5) for $Q(x, z)$.

Had (7) another solution, then (5) would generate another $\hat{Q}(x, z)$ satisfying (7) and (9). As $R^{-}(x, z)^{-1}$ belongs to $L_{1}, \hat{Q}(x, z)$ defined by (5) belongs to $L_{\infty}$. Therefore, the vector $\hat{\mathbf{Q}}(\mathbf{x})$ of coefficients of its Laurent expansion, that satisfies (4) because of (7) and (9), is a column with bounded elements. As matrix $A$ is stochastic, the norm of the operator $x A^{1}$ in the Banach space of columns with bounded elements does not exceed $|x|<1$, so (4) has the only solution in this space. Hence $\hat{\mathbf{Q}}(\mathbf{x})$ and $\mathbf{Q}(\mathbf{x})$ must be equal. This completes the proof of Theorem 1.

Let $q_{0}^{k}$ be the probability of the first return to zero in $k$ steps and $q_{0}(x)=\sum_{k=1}^{\infty} x^{k} q_{0}^{k}-1$.

Corollary. The function $q_{0}(x)$ is expressed by

$$
\begin{aligned}
q_{0}(x)= & (x-1)\left[1+x R^{+}(x, 1) D^{n-1} A_{0}(z) R^{-}(x, z) e(z)\right] \\
& +x \sum_{j=1}^{n-1} q_{j}(x) D^{n-1} A_{0}(z) R^{-}(x, z) T^{+} z^{n-1-j} R^{-}(x, z)^{-1}
\end{aligned}
$$

Indeed, every route of the first return to zero in $k>1$ steps consists of one step to some $j>0$ and then a FZH route from $j$, so

$$
\begin{gathered}
q_{0}^{k+1}=\sum_{j=1}^{\infty} a_{0 j} q_{j}^{k} \quad k>0 \\
q_{0}^{1}=a_{00}=-\sum_{j=1}^{\infty} a_{0 j}+1=\sum_{j=1}^{\infty} a_{o j} q_{j}^{0}+1 .
\end{gathered}
$$

Multiplying both sides of (12) by $x^{k+1}$, summing over $k$ from 1 to $\infty$ and taking into account (13), we obtain

$$
q_{0}(x)=x \sum_{j=1}^{\infty} a_{i j} q_{j}(x)+(x-1)=x D^{0} A_{0}(z) Q(x, z)+(x-1)
$$

which leads to (11) due to (5). 


\section{FZH MEAN TIMES: ORDER OF GROWTH.}

In this section we estimate the order of growth for FZH mean times depending on the number of the starting state. This estimate makes it possible to find $\mu(z)$ by the same method used for $Q(x, z)$, ensuring the existence of all necessary convolutions.

Let $s_{m}$ be the set of states $0,1, \ldots, m ; d^{m}(i, j), i>m, j \leq m$, be the first-descent-to- $s_{m}$ route from $i$ to $j$, and $D^{m}(i, j)$ be the set of all such routes. We introduce functions $N(d)$, the number of steps in the route $d$, and $P R(d)$, the probability of this route. Obviously, if $d$ is a composition of $d_{1}$ and $d_{2}$ then

$$
\begin{aligned}
P R(d) & =P R\left(d_{1}\right) P R\left(d_{2}\right) \\
N(d) & =N\left(d_{1}\right)+N\left(d_{2}\right)
\end{aligned}
$$

Denote

$$
\begin{aligned}
\chi_{i j}^{m} & =\sum_{d \in D^{m}(i, j)} P R(d) \\
\lambda_{i j}^{m} & =\sum_{d \in D^{m}(i, j)} P R(d) N(d) \\
\lambda_{i}^{m} & =\sum_{j=0}^{m} \lambda_{i j}^{m}
\end{aligned}
$$

so that (17) defines the mean length of the first descent from $i$ to $s_{m}$.

Lemma 2.

$$
\mu_{i}=\lambda_{i}^{m}+\sum_{j=1}^{m} \chi_{i j}^{m} \mu_{j} \quad i>m
$$

Proof. Every FZH route from $i$ is $d^{0}(i, 0)$ and obviously is either $d^{m}(i, 0)$ or a composition of $d^{m}(i, j)$ and $d^{0}(j, 0), j=1,2, \ldots, m$. Now (18) readily follows from (16) and (17).

Let us apply (18) to the chain $\left\{\xi_{k}\right\}$ with $n=1$ and denote here $\lambda_{i}$ instead of $\mu_{i}$. For $m=1$ we have here

$$
\lambda_{i+1}=\lambda_{i+1}^{1}+\lambda_{1} \chi_{i+1,1}^{1}
$$


Lemma 3. The FZH mean times for the ergodic Markov chain $\left\{\xi_{k}\right\}$ with a quasitoeplitz transition matrix with $n=1$ satisfy the inequality

$$
\lambda_{i}<\lambda_{i+1}<\lambda_{i}+\lambda_{1} \quad i>0 \text {. }
$$

Proof. Note that for this chain

$$
\lambda_{i}^{m}=\lambda_{i-m}^{0}=\lambda_{i-m} \quad i>m>0
$$

because all the transitions above zero depend on the difference of states. Hence $\lambda_{i+1}^{1}=\lambda_{i}$, so (19) leads to (20).

Now we are back to an arbitrary $n>0$.

Lemma 4. The FZH mean times for the ergodic Markov chain $\left\{\xi_{k}\right\}$ with quasitoeplitz transition matrix do not exceed some linear function of starting state's numbers.

Proof. As the transition matrix is quasitoeplitz, all the transitions above $n-1$ depend on a difference of states, so

$$
\lambda_{i}^{n-1}=\lambda_{i-n+1} \quad i \geq n .
$$

Hence when $m=n-1$ (18) reduces to

$$
\mu_{i}=\lambda_{i-n+1}+\sum_{j=1}^{n-1} \chi_{i j}^{n-1} \mu_{j} .
$$

Note that the sum of coefficients at $\mu_{j}$ on the right side is less than the total probability of first descent to $s_{n-1}$, i.e., less than 1 ; because of $(20)$ $\lambda_{i-n+1}<(i-n+1) \lambda_{1}$. Hence

$$
\mu_{i}<(i-n+1) \lambda_{1}+\sup _{j=1,2, \ldots, n-1}\left\{\mu_{j}\right\}
$$

which proves the statement of Lemma 4.

Let us introduce

$$
\begin{aligned}
\nu_{i} & = \begin{cases}\mu_{i} & i \leq n \\
\mu_{i}-\mu_{i-1} & i>n\end{cases} \\
\nu(z) & =\sum_{i=n}^{\infty} \nu_{i} z^{n-1-i} .
\end{aligned}
$$

Corollary. $\nu(z) \in L_{\infty}$.

Indeed, according to (20) and (21), for all $i>n$

$$
\left|\nu_{i}\right|=\left|\mu_{i}-\mu_{i-1}\right|<\lambda_{1}+2 \sup _{j=1,2, \ldots, n-1} \mu_{j} .
$$




\section{FZH MEAN TIMES: THE GENERATING FUNCTION.}

Theorem 2. The GF $\mu(z),|z|>1$, of the FZH mean times for the ergodic Markov chain $\left\{\xi_{k}\right\}$ with a quasitoeplitz transition matrix with "natural properties" (see [1], pp. 72-73) is defined by the formulas

$$
\begin{gathered}
\mu(z)=z^{2-n} e(z) R^{-}(z)\left[R^{+}(1) e(z)+\sum_{i=1}^{n-1} \mu_{i} T^{+} z^{n-i-2}(z-1) R^{-}(z)^{-1}\right] \\
R^{ \pm}(z)=\exp \left(-T^{ \pm} \ln z e(z)[1-H(z)]\right)
\end{gathered}
$$

where the unknown $\mu_{i}, i=1,2, \ldots, n-1, n>0$, are the only solution of the system of linear equations

$$
\begin{aligned}
& \mu_{i}-\sum_{j=1}^{n-1} \mu_{j} D^{n-2} A_{i}(z) R^{-}(z) e(z) T^{+} z^{n-j-2}(z-1) R^{-}(z)^{-1} \\
& =1+R^{+}(1) D^{n-2} A_{i}(z) e^{2}(z) R^{-}(z) \quad i=1,2, \ldots, n-1, n>0 .
\end{aligned}
$$

Proof. Every FZH or first-return-to-zero route is either one step to zero or consists of one step to some $j>0$ and then a FZH route from $j$. Hence for all $i$

$$
\mu_{i}=\sum_{j=1}^{\infty} a_{i j}\left(\mu_{j}+1\right)+a_{i 0}=\sum_{j=1}^{\infty} a_{i j} \mu_{j}+1
$$

For $i>n-1$ we can rewrite (26) as

$$
\mu_{i}=D^{0} z^{i} H(z) \mu(z)+1 .
$$

According to the definition of $\left\{\nu_{i}\right\}$ we have

$$
\mu(z)=\sum_{i=1}^{n-1} \mu_{i} z^{-i}+z^{2-n} \nu(z) e(z) .
$$

Therefore, multiplying both sides of (27) by $z^{n-1-i}$ and summing over $i$ from $n$ to $\infty$ we obtain

$$
z \nu(z) e(z)=T^{-} z^{n-1} \mu(z) H(z)+e(z) .
$$


Denoting $F^{+}(z)=T^{+} z^{n-1} \mu(z)[1-H(z)]$, due to (28) we have

$$
\nu(z) z[1-H(z)] e(z)=F^{+}(z)+e(z)-\sum_{i=1}^{n-1} \mu_{i} z^{n-1-i}[1-H(z)] .
$$

Note that this is the equality of series, not of their sums, which converge in different areas. $\nu(z)$ is analytic in $\Gamma^{-}$and $F^{+}(z)$ is analytic in $\Gamma^{+}$, though both belong to $L_{\infty}, \nu(z)$ due to the corollary of Lemma 4 , and $F^{+}(z)$ because of Lemma 4 and the absolute convergence of $\sum_{-\infty}^{\infty}|j| h_{j}$, which is one of the required "natural properties." Therefore (29) is a Riemann boundary value problem in $L_{\infty}$.

As was shown in [1], the criterion of ergodicity implies that the functions $R^{ \pm}(z)$ defined by (24) belong to $L_{1}$, so when we multiply both sides of (29) by $R^{+}(z)$ all the convolutions exist and belong to $L_{\infty}$. Then we apply $T^{-}$ to both sides and with the help of Lemma 1 obtain

$$
\nu(z) R^{-}(z)^{-1}=R^{+}(1) e(z)-T^{-} \sum_{i=1}^{n-1} \mu_{i} z^{n-i-2}(z-1) R^{-}(z)^{-1}
$$

which, with (28) yields (23).

The first $n$ equalities of (26) may be written as

$$
\mu_{i}=D^{0} A_{i}(z) \mu(z)+1 \quad i=0,1, \ldots, n-1
$$

from which, with the use of (23), we obtain (25).

Suppose the system (25) has a solution other than $\mu_{i}, i=1,2, \ldots, n-1$, say $\hat{\mu}_{i}$. Then (23) would generate another sequence $\hat{\mu}_{i}, i=0,1, \ldots$, such that the vector $\Delta M=\left\{\hat{\mu}_{i}-\mu_{i}\right\}^{T}$ would be bounded (as $R^{-}(z)^{-1}$ and $R^{-}(z)$ belong to $L_{1}$ ).

Obviously the sequence $\left\{\hat{\mu}_{i}-\mu_{i}\right\}$ satisfies the equations

$$
\left(\hat{\mu}_{i}-\mu_{i}\right)=\sum_{j=1}^{\infty} a_{i j}\left(\hat{\mu}_{i}-\mu_{i}\right) \quad i=0,1, \ldots
$$

Let us multiply both sides of (30) by $p_{i}$, which is the stady state probability of state $i$, and sum over $i$ from 0 to $\infty$. With $\Delta M$ bounded all the sums present converge absolutely, so we can change the order of summation and make use of the equilibrium equations

$$
p_{j}=\sum_{i=0}^{\infty} p_{i} a_{i j} \quad j=0,1, \ldots
$$


to obtain

$$
\sum_{i=0}^{\infty} p_{i}\left(\hat{\mu}_{j}-\mu_{j}\right)=\sum_{i=0}^{\infty} p_{i} \sum_{j=1}^{\infty} a_{i j}\left(\hat{\mu}_{j}-\mu_{j}\right)=\sum_{i=0}^{\infty} p_{i}\left(\hat{\mu}_{i}-\mu_{i}\right)-p_{0}\left(\hat{\mu}_{0}-\mu_{0}\right) .
$$

Due to the conditions of descent (see [1]) $p_{0}>0$, so $\hat{\mu}_{0}=\mu_{0}$. Thus we can rewrite (30) as $\Delta \boldsymbol{M}=\boldsymbol{A} \boldsymbol{\Delta} \boldsymbol{M}$. According to Chung [5], this means that $\Delta \boldsymbol{M}$ must be collinear with $(1,1, \ldots)^{T}$, so with $\hat{\mu}_{0}-\mu_{0}=0, \Delta \boldsymbol{M}$ must be zero. This concludes the proof of Theorem 2.

Corollary. The mean time of the first return to zero is

$$
\begin{aligned}
\mu_{0}= & 1+R^{+}(1) D^{n-2} A_{0}(z) e^{2}(z) R^{-}(z) \\
& +\sum_{i=1}^{n-1} \mu_{i} D^{n-2} A_{0}(z) R^{-}(z) e(z) T^{+} z^{n-i-2}(z-1) R^{-}(z)^{-1} .
\end{aligned}
$$

This follows, with the use of (23), from (26) with $i=0$ written as

$$
\mu_{0}=D^{0} A_{0}(z) \mu(z)+1 \text {. }
$$

\section{DUALITY.}

In conclusion we discuss the relationship of duality between transient and steady state probabilities from one side, and FZH probabilities and mean times increments, respectively, from the other. This relationship is imposed in a natural way by the method of Riemann boundary value problems.

Following [4] we consider two Riemann boundary value problems

$$
\begin{aligned}
& a_{1}^{+}(z) K_{1}(z)=a_{1}^{-}(z)+b_{1}(z), \quad z \in \Gamma \\
& a_{2}^{+}(z) K_{2}(z)=a_{2}^{-}(z)+b_{2}(z), \quad z \in \Gamma
\end{aligned}
$$

dual if and only if

(a) Banach spaces where solutions must be found for these problems are clual;

(b) $K_{1}(z) K_{2}(z)=1$. 
When the dual problems have unique solutions, their duality imposes the duality of these solutions. We can easily see that with this in mind the GF's of the transient state probabilities and FZH probabilities are dual. Indeed, they belong to the dual Banach spaces $L_{1}$ and $L_{\infty}$, and the coefficients of their respective boundary value problems (see (10) in the present paper and (15) in [1]) are $[1-x H(z)]$ and $[1-x H(z)]^{-1}$, respectively.

The same duality exists between the steady state probabilities and the sequence of increments of FZH mean times. Their GF's belong, as was proved above, to $L_{1}$ and $L_{\infty}$, respectively. The coefficients of their respective boundary value problems are $[1-H(z)] z e(z)$ and $\left(1-z^{-1}\right)[1-H(z)]^{-1}$.

\section{REFERENCES.}

1. Dukhovny, A.M. (1989) Markov Chains with Quasitoeplitz Transition Matrix, Journal of Applied Mathematics and Simulation, 1:.

2. Spitzer, F. (1956) A Combinatorial Lemma and its Applications to Probability Theory, Trans. Amer. Math. Soc., 82: 323-339.

3. Dukhovny, A.M. (1982) A One-Dimensional Random Walk that Depends on a Parameter, Dokl. Akad. Nauk. Ukrain. SSR, Seria A. 3:9-12.

4. Gahov, F.D., Boundary Problems. Fizmatgiz, Moscow, 1964.

5. Chung Kai-lai, Markov Chains with Stationary Transition Probabilities, Springer-Verlag, New York, 1967. 


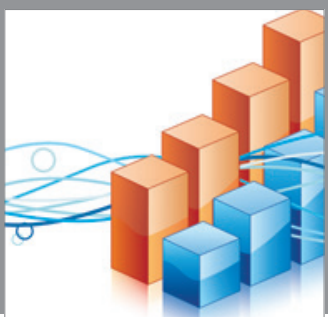

Advances in

Operations Research

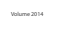

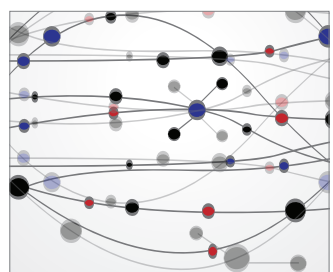

\section{The Scientific} World Journal
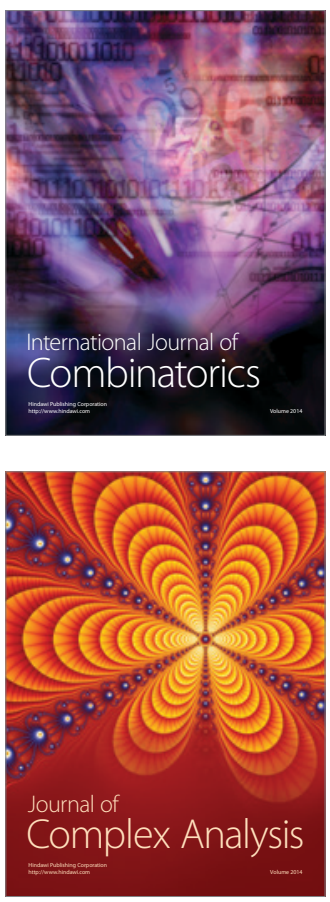

International Journal of

Mathematics and

Mathematical

Sciences
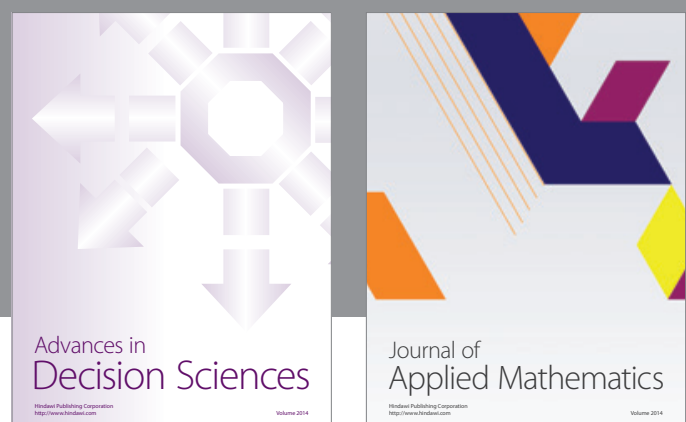

Journal of

Applied Mathematics
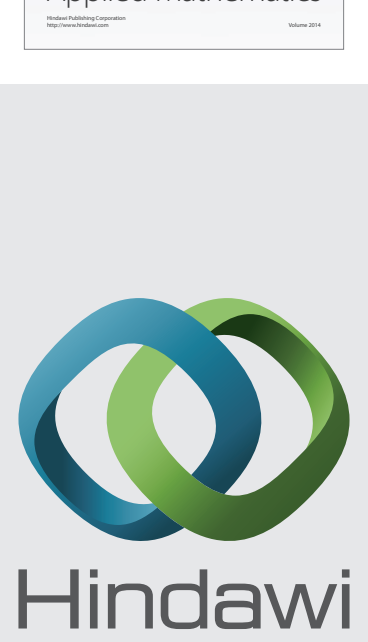

Submit your manuscripts at http://www.hindawi.com
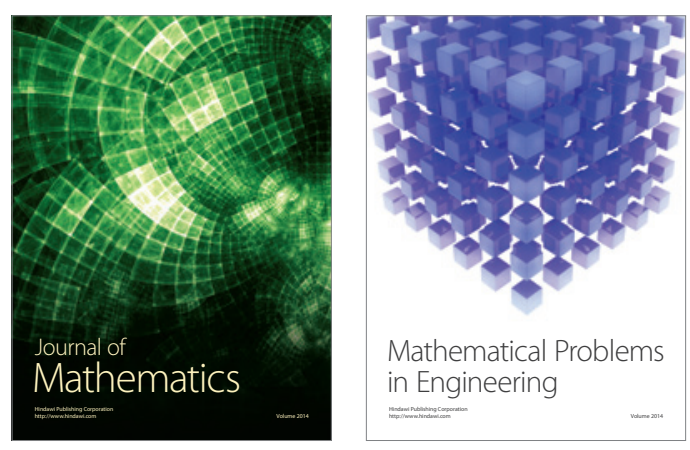

Mathematical Problems in Engineering
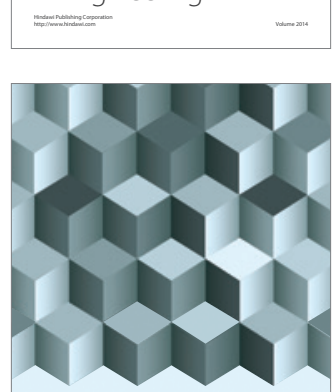

Journal of

Function Spaces
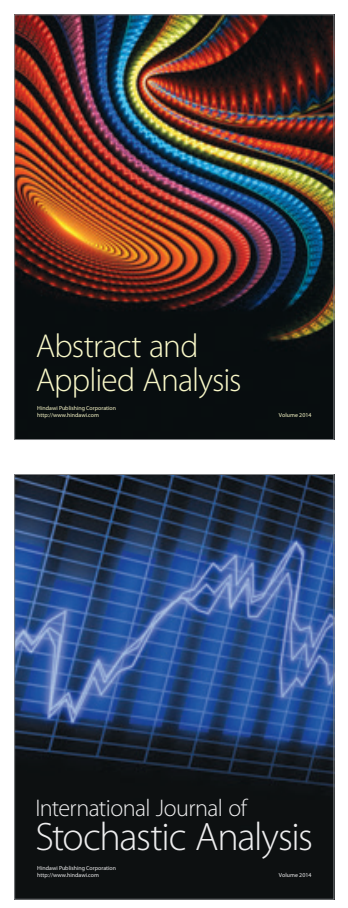

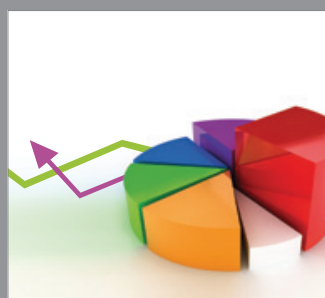

ournal of

Probability and Statistics

Promensencen
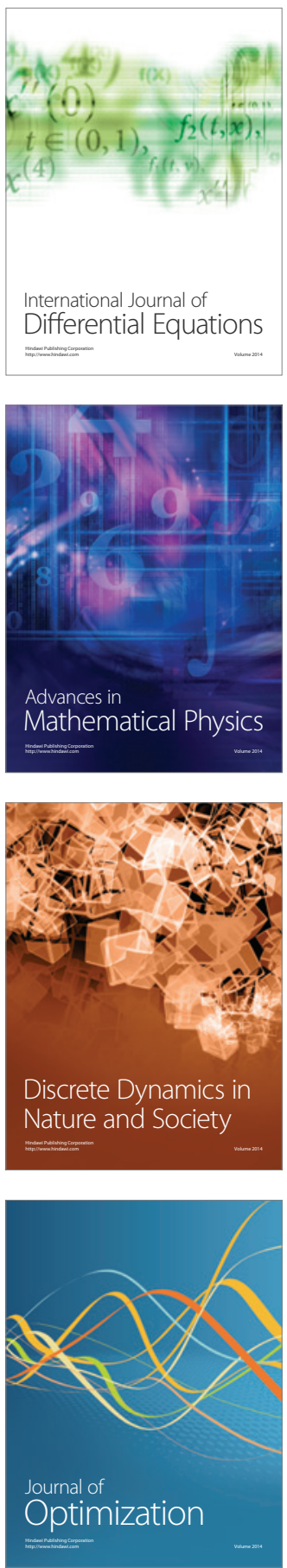